Service social

\title{
L'approche féministe en termes de rapports sociaux ou l'art de survivre sur la corde raide en talons hauts!
}

\section{Michèle Bourgon}

Volume 36, numéro 2-3, 1987

Approches intégrées

URI : https://id.erudit.org/iderudit/706362ar

DOI : https://doi.org/10.7202/706362ar

Aller au sommaire du numéro

Éditeur(s)

École de service social de l'Université Laval

ISSN

1708-1734 (numérique)

Découvrir la revue

Citer cet article

Bourgon, M. (1987). L'approche féministe en termes de rapports sociaux ou l'art de survivre sur la corde raide en talons hauts! Service social, 36(2-3), 248-273. https://doi.org/10.7202/706362ar
Résumé de l'article

Préoccupée d'une part par la situation des femmes qui, suite aux bouleversements provoqués par les luttes féministes, ont à vivre sous la pression de messages sociaux contradictoires, intéressée d'autre part par les pratiques sociales qui, dans le contexte actuel de transformations basées sur la rationalisation des budgets et des services, se trouvent confrontées à des visions divergentes quant à la nature et la fonction de l'intervention sociale, Pauteure expose et illustre une approche d'intervention féministe visant la défense des intérêts des femmes et la transformation des rapports sociaux dominants. 
BOURGON, Michèle, professeure au Département de travail social de l'Université du Québec à Montréal.

\section{L’approche féministe en termes de rapports sociaux}

ou

l'art de survivre sur la corde raide en talons hauts!*

\section{Michèle Bourgon}

\section{Un défi de taille à relever...}

Deux préoccupations majeures ont motivé la rédaction de ce texte : la situation des femmes en 1988 et l'état actuel des pratiques en travail social au Québec.

\section{Quand le rose tire encore au gris}

La preuve n'est plus à faire. Malgré les progrès qu'ont amenés les grandes luttes féministes des vingt dernières années, la majorité des femmes sont encore privées de beaucoup de ressources nécessaires pour être en bonne santé physique et mentale. ${ }^{1}$ Tout ceci dans un contexte idéologique où elles reçoivent de plus en plus de messages contradictoires lourds et "vidants " à gérer. En effet, d'un côté, elles sont incitées à l'expression d'elles-mêmes, à l'épanouissement de leur personne, à l'affirmation de leurs besoins, à l'amour d'abord et avant tout de soi; d'un autre côté, on leur demande, et ce de façon tout aussi pressante, de se sentir encore pratiquement les seules et uniques responsables du bien-être physique et psychologique de leur mari et de leurs enfants.

Est-il surprenant alors que tant de femmes craquent sous de telles pressions conflictuelles? Une recherche que nous avons menée récemment en collaboration avec le Y.W.C.A. de Montréal ${ }^{2}$ laisse présager 
que les vingt prochaines années ne seront pas, elles non plus, de tout repos. On commence à peine par exemple, à examiner et à connaître les effets immédiats et futurs de l'entrée massive des femmes sur le marché du travail salarié. À quel prix, pour leur santé et celle de leurs enfants présents et futurs, s'est faite cette intégration au monde masculin de la production qui, lui, a quand même très peu changé ? Et que dire de ces nouvelles technologies de la reproduction que nous vantent sans presque aucun sens critique les média depuis quelques temps ? ${ }^{3}$ II existe un danger réel que les femmes deviennent les cobayes d'expériences "scientifiques" dont on connaît finalement peu les effets à long terme sur leur qualité de vie et celle de leurs enfants; leur corps risque de devenir une machine de procréation sur laquelle elles auront peu ou pas de pouvoir. Les effets du vieillissement de la population auront également pour elles des conséquences très lourdes.

Les retombées de ces bouleversements concernent notre profession de près. D'abord parce que la majorité d'entre ses membres auront à les vivre en tant que femmes, dans leur vie personnelle, et ensuite parce que, dans de telles conditions, la majorité de notre "clientèle ${ }^{4}{ }^{\text {risque }}$ fort de demeurer à composition féminine pour plusieurs années à venir...

\section{Le contexte de la pratique actuelle... un numéro de corde raide}

Mais qu'en est-il exactement du contexte de la pratique en travail social, en 1988 ? Au lendemain de la publication du Rapport Rochon et du Rapport Harnois, le champ de l'intervention sociale se trouve sur des sables mouvants. Dans une conjoncture de transformations majeures où les mots de passe sont " compressions budgétaires", "rationalisation des services", "désinstitutionnalisation " et "transferts d'effectifs", les intervenantes sociales des réseaux publics et para-publics se retrouvent dans une situation qui est pour le moins périlleuse. Confrontées quotidiennement à des visions contradictoires sur la nature et la fonction de leurs interventions, ${ }^{5}$ elles ont continuellement à assumer les retombées que ces contradictions génèrent. Pensons par exemple à l'intervenante, formée par ses professeurs à la relation d'aide, appelée à intervenir dans un contexte d'autorité où son employeur s'attend à ce qu'elle contrôle et surveille, et où le "bénéficiaire" s'attend à ce qu'elle règle un problème matériel et lui foute la paix ! Ce genre de situation place les intervenantes sur une corde raide d'où il est dangereux de tomber. Car d'une part les attend le missionnariat-sacrifice qui implique qu'elles doivent se battre sans arrêt et sans relâche contre les règles d'un 
système qui vont à l'encontre de tout ce qu'elles veulent mettre de l'avant; et d'autre part, l'acceptation et l'identification à ces mêmes règles, au prix de faire taire leurs convictions profondes... Assez ironiquement, tomber de l'un ou l'autre côté de cette corde raide peut mener à peu près au même résultat : saper l'énergie des intervenantes et aboutir à un burn-out. ${ }^{6}$

Comment, dans un tel contexte si objectivement difficile, obtenir pour les femmes qui se retrouvent volontairement ou pas dans les bureaux des travailleuses sociales, les ressources dont elles ont si grandement besoin, sans pour autant "se brûler " à le faire ? Ou, dit autrement, comment nous, en tant que praticiennes du social, allons-nous mener des interventions qui défendent les intérêts des femmes et visent la transformation des rapports sociaux dominants sans tomber de la corde raide sur laquelle nous place actuellement le contexte de la pratique au Québec?

Cet article veut tenter de répondre à ces questions en présentant une façon alternative de travailler avec les femmes et les jeunes filles qui tient compte du contexte que nous venons de décrire ${ }^{7}$ et sans pour autant sacrifier les grandes visées du mouvement féministe. En présentant le modèle de pratique en termes de rapports sociaux que nous développons depuis quelques années, nous espérons contribuer à relever le défi auquel ont à faire face tous ceux et toutes celles qui œuvrent dans le domaine du travail social : celui de développer des analyses et des pratiques qui reconnaissent et agissent sur les conflits socio-politiques et idéologiques actuellement présents dans le champ social au Québec et qui se retrouvent reproduits, "veut-veut pas ", à petite échelle entre les quatre murs des bureaux des C.S.S., C.L.S.C. et groupes autonomes.

Pour arriver à dégager en quoi consiste notre modèle à ce momentci de son développement, nous procéderons de la façon suivante. D'abord nous en présenterons brièvement les prémisses, l'objectif et les principales considérations méthodologiques. Nous illustrerons ensuite comment ces éléments se traduisent dans la pratique en présentant, à titre d'exemple, des extraits commentés d'une première entrevue avec une jeune fille de 14 ans. Et, finalement, nous identifierons deux tâches importantes qui attendent, selon nous, celles qui veulent intervenir à partir d'une perspective féministe en travail social. 


\section{Présentation du modèle}

\section{Les prémisses}

Trois prémisses sous-tendent en ce moment l'approche féministe en termes de rapports sociaux. La première est que les sentiments, les pensées (les idéologies) et les actes d'une personne découlent des ressources dures et molles auxquelles elle a eu accès en tant que membre d'une collectivité identifiée comme ayant un sens spécifique dans une société donnée (ex. la collectivité des hommes, celle des noirs, celle des plus de 65 ans, etc.) Cette prémisse va à l'encontre du mythe particulièrement tenace au sein de notre société qui cherche à nous faire croire qu'un "individu" a en lui toutes les ressources dont il a besoin pour être "heureux" et qu'en finalité, lui seul est l'ultime responsable de sa situation. Elle va aussi à l'encontre de la façon dichotomisante avec laquelle la plupart des autres modèles en travail social analysent en ce moment une situation en voyant l'individu (l'intérieur) d'un côté et le contexte (l'extérieur) de l'autre. Cette prémisse suggère plutôt que l'acteur ou l'actrice sociale hors de son contexte n'existe tout simplement pas ${ }^{8}$.

Une seconde prémisse sous-tend également cette approche : que l'accès aux ressources est déterminé par les structures politiques, économiques et idéologiques auxquelles donnent naissance les rapports sociaux actuels. Cette prémisse, pour sa part, questionne les mythes dominants de la "méritologie " et du volontarisme qui font que tous les individus agissent par choix, peuvent changer par volonté et donc récoltent ce qui leur arrive par mérite.

Une troisième prémisse découle des deux précédentes : seule une transformation en profondeur des rapports sociaux dominants et des structures actuelles qui en résultent peut mener à une véritable modification des règles du jeu du présent système. Ces structures établies sous les rapports sociaux du patriarcat et du capitalisme sont sexistes (discriminatoires à l'égard des femmes), " classistes » (discriminatoires à l'égard des personnes de classe populaire) et racistes (discriminatoires à l'égard des personnes non blanches). Elles sont également hétérosexistes (discriminatoires à l'égard des gais et des lesbiennes), " âgistes " (discriminatoires à l'égard de ce que notre société a défini comme étant les "jeunes"-en ce moment les moins de 30 ans - et les "vieux": les plus de 50 ans chez les hommes, les plus de $\mathbf{4 0}$ ans chez les femmes) et "handicapistes" (discriminatoires à l'égard des personnes qui ont un handicap physique - "les infirmes" -, psychologique - "les fous" ou mental - "les débiles" -). 
Ceci veut dire par exemple qu'au Québec en 1988 - et il est important ici de garder en tête la notion d'historicité d'Alain Touraine - un homme blanc de 35 ans, hétérosexuel et de classe bourgeoise est considéré comme possédant plus de valeur sociale qu'une femme noire de 65 ans vivant sur le B.E.S. Cette valeur sociale, accordée indépendamment des acteurs en place, permet à l'homme d'avoir davantage accès à de meilleurs services médicaux : s'il a le cancer, il connaît les meilleurs spécialistes et peut choisir, pas elle. Il a également accès à de meilleures conditions d'habitation, dans des quartiers moins pollués, et a l'attention des serveurs et serveuses dans les restaurants. Bref, il a accès à toutes sortes de ressources dures et molles qui contribuent à le garder en bonne santé mentale et physique selon les normes de cette même société. Il peut cependant refuser certains des privilèges que lui confère "veut-veut pas » son statut d'homme blanc et bourgeois. Il peut, par exemple, refuser de demeurer dans un quartier comme Westmount et choisir plutôt Saint-Henri, où il luttera avec d'autres locataires afin d'y améliorer les conditions de logement. ${ }^{9}$ - II ne peut, par contre, changer le fait qu'un policier, qui est comme nous tous le produit de cette société "classiste", sexiste et raciste, aura tendance - à moins qu'il ne s'avise et agisse de façon consciente sur les manifestations de son racisme / classisme"/ sexisme - à accorder plus de poids, plus de crédibilité et plus d'importance aux propos d'un homme blanc, jeune et bourgeois qu'à ceux d'une femme noire, âgée et pauvre. Cet état de fait, qui peut sembler de l'ordre de l'évidence, est cependant rarement pris en compte lorsqu'il s'agit de comprendre la manière d'être, de penser et d'agir d'une personne quand cette dernière se retrouve dans le bureau d'une travailleuse sociale. On se refère alors, plus souvent qu'autrement, à des théories explicatives qui coupent la personne de son contexte - la fameuse dichotomie dont nous parlions plus haut - et cherchent en elle les raisons de ses comportements, de ses sentiments et de ses idées.

À ces trois prémisses fondamentales s'ajoute un constat dont la preuve, à notre avis, n'est plus à faire : celui de reconnaître que les règles matérielles et idéologiques du présent système placent toutes les femmes dans des situations intenables - pensons entre autres au choix que de plus en plus d'entre elles doivent faire : avoir ou non des enfants - et privent en même temps la majorité d'entre elles des ressources dont elles ont besoin pour s'en sortir, par exemple, en les gavant, dès le berceau, de culpabilité, l'arme suprême de la soumission. 


\section{L'objectif principal}

De ces prémisses et de ce constat découle le seul objectif que peut, que doit avoir une intervention féministe en travail social : obtenir pour et avec les femmes avec lesquelles nous travaillons les ressources dont elles disent avoir besoin pour changer les situations difficiles dans lesquelles l'état actuel des rapports sociaux les place.

\section{Quelques considérations méthodologiques}

Les prémisses et l'objectif que nous venons de présenter entraînent certaines considérations méthodologiques. Parmi celles-ci, mentionnons celle qui demande de voir le rapport d'intervention non pas comme une relation d'aide, une relation égalitaire, ou un rapport de service 10 entre deux ou plusieurs individus qui vise à aider, à lutter ou à servir, mais plutôt comme un rapport politique entre acteurs et actrices sociales au sein duquel s'affrontent des visions idéologiques contradictoires et dont le but est de gérer les conflits qui découlent de l'état actuel des rapports sociaux.

La figure qui suit illustre les visions idéologiques contradictoires qui peuvent exister au sein du rapport d'intervention autour du but et des rôles des parties impliquées. Si l'intervenante ne reconnaît pas que sa vision des composantes du rapport d'intervention et celle de "l'usagère" sont le produit de leurs positions respectives au sein de la société et plus particulièrement au sein d'un organisme du travail social et que, de plus, elle impose sa vision, si féministe soit-elle, du but de l'intervention, elle reproduit, sur une petite échelle, un rapport de domination d'une classe sur une autre et ce faisant, nuit à celle qu'elle prétend aider. Si par contre l'intervenante reconnaît que les idéologies en place - $y$ inclus celles véhiculées par l'organisme - sont le reflet de positions politiques conflictuelles, elle peut les rendre explicites et les utiliser pour questionner les règles du jeu. Ceci peut être fait par exemple en questionnant le mythe de l'expert bienveillant dont le savoir objectif et scientifique lui permet de porter un jugement "neutre" sur n'importe quelle situation et sur n'importe quelle personne. 
Rapports sociaux dominants et dominés

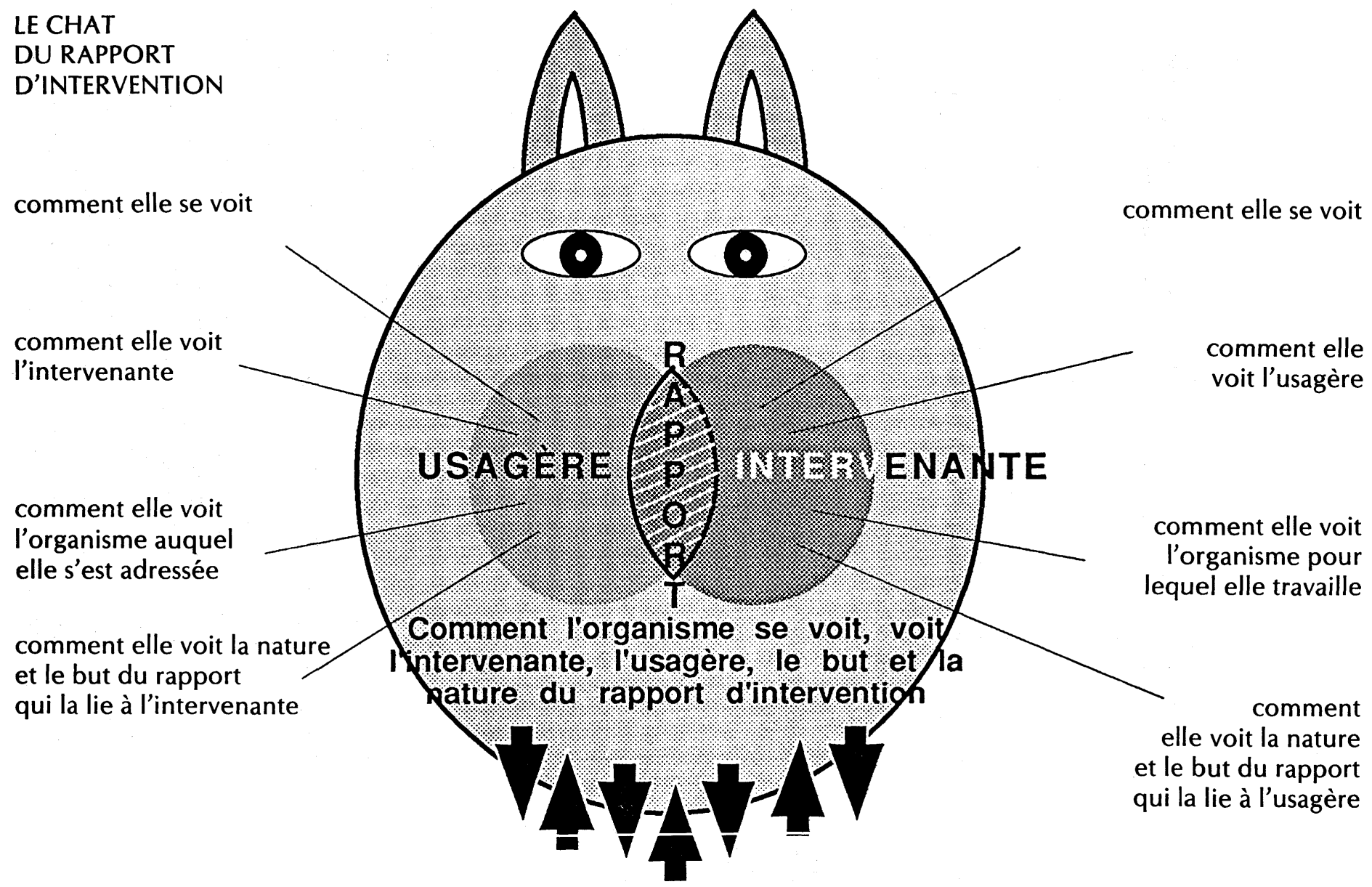


Les prémisses et l'objectif décrits plus haut ont une deuxième conséquence pour la pratique: celle de reconnaitre qu'il doit, non seulement exister plusieurs niveaux complémentaires d'intervention, mais que ces niveaux doivent s'inspirer d'une perspective d'analyse commune si l'on veut que les ressources que l'on développe pour et avec les femmes leur permettent de changer leur situation. Ceci veut dire que toutes les interventions, peu importe le nombre de personnes impliquées, doivent être menées de manière à :

1. déculpabiliser et "dévictimiser" les femmes, car elles ne sont ni coupables (de l'intérieur) ni victimes (de l'extérieur) des situations qu'elles vivent. Elles sont plutôt le produit des ressources auxquelles elles ont eu accès en tant que femmes dans l'état actuel des rapports sociaux;

2. laisser aux femmes le soin de nommer leur réalité de la façon qu'elles la vivent plutôt que de leur imposer une vision "professionnelle", "technocratique" ou "militante" de cette même réalité. Intervenir de façon à replacer leurs propos, les nôtres et ceux de l'organisme au sein duquel nous nous rencontrons dans le contexte plus large qui leur donne tout leur sens et toute leur signification;

3. éviter de personnaliser les conflits idéologiques en jeu, par exemple : "elle manque d'insight " ou encore " elles ne sont pas assez conscientisées ". Utiliser la présence de ces conflits pour questionner la nature du rapport d'intervention vu comme rapport social et point de départ pour interroger l'organisation même de notre société.

\section{Rencontre avec Mélanie}

L'entrevue qui suit illustre comment les prémisses, le but et les considérations méthodologiques que nous venons de présenter se concrétisent dans la pratique. ${ }^{11} \mathrm{II}$ s'agit d'une première rencontre avec Mélanie, une jeune fille de 14 ans qui a fugué de chez elle pendant trois jours et qui, à son retour à la maison, se voit obligée par ses parents à voir une travailleuse sociale.

L'objectif que vise l'intervenante pour cette première entrevue: élaborer un contrat de travail qui établisse les paramètres de l'intervention tout en tenant compte de la nature du rapport entre les actrices en place et du contexte plus large dans lequel ce rapport s'inscrit. Toutes les questions que pose l'intervenante se situent uniquement dans cette perspective. Cette dernière ne cherche pas à comprendre à ce momentci par exemple, pourquoi Mélanie a voulu quitter la maison, parce que cette question n'est pas pertinente à l'atteinte du but. Par contre, 
aborder ce point pourrait devenir très opportun dans les deux entrevues subséquentes où il sera question, comme nous le verrons, d'une décision possible de quitter ses parents.

Nous avons choisi cette phase spécifique de l'intervention pour illustrer nos propos parce que nous croyons que l'établissement du contrat qui doit s'y effectuer est un moyen privilégié pour amorcer une transformation du rapport professionnel.

I. Bonjour Mélanie. Mon nom est Jacinthe Latour [nom fictif]. J'suis travailleuse sociale ici et c'est moi qui ai reçu ton dossier. La secrétaire m'l'a remis ce matin, alors j'viens tout juste de l'regarder. Et c'est pour ça que c'est moi qui suis ici. Parce que c'est moi qui ai reçu ton dossier... moi?

M. Pourquoi j'suis ici

I. C'tune bonne question ça. Tu t'demandes pourquoi t'es ici...

M. Ouain! Ma mère m’a dit qu'y fallait quej'vienne mais personnem'a dit pourquoi.

I. OK. Moij'peux t'montrer l'information que j'ai ici. Tout ce que j'sais d'toi et de pourquoi t'es ici, c'est c'qu'il y a d'écrit sur ce bout de papier. Nous pouvons l'regarder ensemble et de cette façon-là on va avoir toutes deux l'même niveau d'information. Tu vois c'est écrit ici que t'es partie d'la maison pendant trois jours et que ta mère est inquièteà ton sujet et qu'elle veut que tu voies une travailleuse so-
L'intervenante commence son entrevue en partageant avec Mélanie toute l'information qu'elle possède à son sujet. Elle sait qu'il existe une relation de pouvoir entre elle et l'adolescente, qu'elle le veuille ou non. En tant que professionnelle au sein d'un organisme social, elle a le statut et le pouvoir que lui confèrent son rôle et sa fonction.

Elle est plus âgée que Mélanie, a plus d'instruction et vient probablement d'un milieu socio-économique plus " privilégié " que le sien. Elle a donc accès à plus de ressources matérielles et affectives et possède, aux yeux de notre société, plus de "valeur sociale" que la jeune fille. $\mathrm{Si}$, en plus, l'intervenante était un homme, elle aurait toutes les bonnes cartes en main. Ces différences et le pouvoir qu'elles impliquent sont rarement prises en compte au sein du rapport d'intervention. L'intervenante, dans la présente situation, essaiera d'en tenir compte. Elle encouragera Mélanie à utiliser leur relation pour questionner l'immuabilité des règles sociétales en lui offrant l'expérience d'un autre type de rapport que ceux auxquels elle est habituée dans sa vie de tous les jours. Même si c'est la première fois que Mélanie rencontre une travailleuse sociale, elle connaît déjà les règles du jeu puisque ces dernières sont, à peu de chose près, les mêmes que celles qu'elle a connues dans sa vie jusqu'à maintenant. Elle sait, qu'en tant que jeune, elle n'a pas vraiment le 
ciale parce qu'elle pense que t'as des problèmes. C'est tout ce que j'ai comme information en ce moment.

M. Luiavez-vousparlé à ma mère, vous ?

I. Non j'lui ai pas parlé directement. Elle a donné ces renseignements à la femmequi travailleàl'accueil. C'est elle qui prend tous les appels ici au C.S.S. Tout ce que j'sais c'est ce qu'il y a d'écrit sur cette feuille de papier. Est-ce que ta mère t'a dit qu'elle avait téléphoné à notre bureau ? Est-ce qu'elle t'a dit pourquoi tu venais voir une travailleuse sociale?

M. Ben, a m'l'a dit aujourd'huiquandj't'arrivée de l'école...

I. Est-ce qu'elle t'a expliqué pourquoi tu devais venir ici?

M. Pas vraiment. Am'a dit "Tu y vas, un point c'est tout. T'as pas l'choix. "J'y ai dit "pourquoi faut que j'aille? " Pis a m'a dit : "Faut que t'ailles. Ton père $t^{\prime} l e$ dira pourquoi. " Pis là mon pèrey'a pas dit grand chose, seulement qu'y fallait que j'viennesinon y'm'placerait dans un Centre ou quelque chose comme ça. Y'a ditque j'tais folle... hein?

I. C'est "heavy» ça

M. Ouain... Y'est capoté ben raide. $Y$ pense que sa fille devrait être la sainte pouvoir de définir sa réalité. On lui dira ce qu'elle a, qui elle est et ce qu'elle doit devenir, c'est-à-dire une bonne fille. La compréhension qu'elle a de sa situation et l'interprétation qu'elle donne au fait de se retrouver dans le bureau d'une travailleuse sociale sont rarement considérées comme étant une analyse valable - quoique différente - de ce qui se passe. Ses propos seront plutôt filtrés à travers des prismes " agéistes ", " classistes » et sexistes qui font qu'on ne tiendra pas compte de ce qu'elle dit parce que, comme tout le monde le sait, "les filles exagèrent et manipulent ". C'est de cette façon que le rapport d'intervention en travail social peut reproduire les règles qui découlent des rapports sociaux actuels. Mais ce rapport peut également servir à questionner ces mêmes règles et ces mêmes rapports sociaux.

Voyons comment l'intervenante le fait ici avec Mélanie. Tout d'abord, elle croit la jeune fille quand cette dernière lui dit ne pas savoir pourquoi elle se retrouve dans le bureau d'une travailleuse sociale. Elle lui reconnaît le droit d'en connaître les raisons exactes. Puisqu'il est dans son pouvoir dans ce cas-ci de le faire, l'intervenante donne à la jeune fille accès à toute l'information qu'elle possède. Elle ne lui cache pas certains faits afin d'être en mesure de mieux la piéger plus tard et de lui faire admettre ses torts, comme ça se passe souvent dans de telles situations. Elle partage plutôt l'information afin que la jeune fille sache exactement où elle en est et à qui elle a affaire. Tout au long de ce passage de l'entrevue, l'intervenante vérifie auprès de Mélanie quels sentiments et quelles pensées provoque chez elle cette visite à une travailleuse sociale. Elle reconnaît ouvertement que d'avoir été 
vierge en personne. /'suis la seule fille dans la famille, j'peux rien faire. Même pas sortir...

I. Hum hum... Pis c'est comme si le fait que t'aies été obligée de venir ici cet après-midi, c'est une autre preuve que tu peux rien faire. Que moij'sus supposée de... Est-ce que tes parents t'ont dit ce que moi j'devais faire exactement?

M. Y'ont juste dit que j'étaisfuckée pis queje devais venir vous voir c'est tout...

I. As-tu l'impression que j'sus supposée " t'arranger ", te mettre sur le bon chemin?

M. Ouais. c'est ça. Y'ont ditquej'étaisfuckée pisqu'y veulent que j'soye comme eux autres y veulent que j'soye. I'pensais que vous seriez comme eux.

I. Tu devais pas avoir ben ben hâte de m'voir eh? (rire)

M. Non.

I. Sic'est ça que tu pensais, tu devais te demander cequej'allais faire là-dedans, cequej'allais penser de tout ça?

M. Ouain... je pensais que t'allais m'faire un sermon...

I. C'est ça que tu pensais que j'allais faire?

M. C'est ben sûr. De toute façon, avecles adultes c'est toujours pareil. Essaye obligée de se présenter à ce bureau sous "pression psychologique" - menaces de l'envoyer dans un centre - a dû être frustrant et difficile à vivre. Elle évite ainsi d'interpréter des réserves fort justifiées de la part de l'adolescente, comme des preuves de "résistance au changement" et/ou de "manque de motivation "... Mélanie a toutes les raisons du monde d'être méfiante à l'égard de l'intervenante. Cette dernière a le pouvoir de prendre des décisions qui peuvent bouleverser sa vie. L'intervenante rendra explicite ce pouvoir lorsqu'elle décrira l'agence pour laquelle elle travaille.

Dans ce court passage, l'intervenante rend explicite le rôle que doit habituellement jouer une travailleuse sociale, c'està-dire celui de "redresser " les déviants et les déviantes du droit chemin. En faisant ceci, elle donne une voix et une crédibilité aux appréhensions de l'adolescente. 
d'leur parler voire... Tu peux pas; y sont toujours en train de faire des sermons.

I. Est-cequej'suisà peu près del'âge de tes parents?

M. Ouain...

SILENCE pire...

Mais tu sembles pas si

I. Comment ça ?

M. Ben,tum'as pas crié après...

I. (Rire) T'avais-tu peur de ça aussi ?

M. Ben, j'sus toujours en train de m'faire crier après...

I. T'aimerais-tu ça savoir c'que je fais exactement Mélanie ? J'ai comme l'impression que ça t'dérange un peu. Tu t'dis «a vas-tu me crier après ? a vas-tu me faire dessermons ? a vas-tu m'envoyer dans un Centre ?..."

M. Ouain... c'est comme si j'avais rien à dire dans ma vie. Envoye paricitte, envoye parlà. C'est toujours euxquidécident tout. Ypeuvent m'envoyer à un... en quelque place pisj'auraipas un mot à dire là-d'dans.

I. C'est ce qui est arrivé aujourd'hui, eh?

M. Exact.
Mélanie et l'intervenante savent très bien qu'il existe une grande différence d'âge entre elles. En soulignant ce fait ouvertement - " je suis une adulte du même âge que tes parents " I'intervenante reconnaît que les paramètres de sa relation avec la jeune fille sont définis par le monde "extérieur " et que la société tout entière est présente dans ce petit bureau. De cette façon, elle va à l'encontre du mythe qui prétend que le rapport professionnel est dénué de telles considérations " terreà-terre" et que "l'aidant " est un esprit désincarné dont la seule fonction est d'aider sa " cliente " à atteindre un niveau plus élevé de bien-être psychologique.

Une fois de plus, l'intervenante rend explicites les différentes perceptions qui existent autour de son rôle. Mélanie a toutes les raisons de croire qu'elle va la "sermonner" car c'est en effet ce que font la plupart des adultes, et les travailleurs sociaux ne font pas souvent exception à la règle!

Ici l'intervenante resitue l'impuissance que vit Mélanie face à sa présence dans ce bureau, au sentiment d'impuissance plus large qu'elle vit en général. 
I. OK. Ben écouteMélanie,j'peux t'expliquer c'que j'fais ici et pis toi, si t'as des questions sur c'que j'dis, tu m'les demandes OK? T'as pu remarquer que j'sus pas mal plus vieille que toi pis t'as raison, des fois on pense pas pareil toi pis moi. Fait qu'y faut me l'dire quand c'quej'disc'est pas clair pour toi. Ça va-tu ça ?

\section{Ouain...}

I. Ben moi j'travaille pour une agence qu'y s'appelle le C.S.S.M.M. On fait ben des choses... on fait des adoptions, on trouve des places pour des personnes âgées, on travaille dans les hôpitaux. Pis on prend aussi des appels de parents et d'enfants qui sont en difficulté.

Quand nous jugeons, quand j'juge qu'une enfant est en danger, c'est-à-dire qu'elle peut se faire mal ou faire mal à des gens autour d'elle ou alors qu'a peut se faire blesser par eux, ben alors mon service y intervient. Nous pouvons effectivement enlever une enfant à sa famille si nous jugeons que la situation est assez grave pour ça. $\mathrm{Ou}$ on peut demander qu'une famille vienne voir une travailleuse sociale pendant un certain temps. Ou encore on peut suggérer d'autre chose...

INTERROMPT 
M. Alors comment ça s'fait qu'y sont pas ici mon père pis ma mère? Comment ça s'fait que c'est juste moi qui es ici?

I. O.K. C'est une bonne question ça aussi. La seule raison que toi t'es ici en ce moment c'est que c'est ta mère qui a téléphoné et qui a dit " jeveux que vous voyez ma fille. À s'est sauvée de la maison ". Pis que toi, tu t'es présentéeà ton rendez-vous cet après-midi. elle?

M. Allez-vous la voir,

I. Ben ça ça dépend de c'qu'on va décider ensemble cet après-midi. Le seul but de notre rencontre aujourd'hui, Mélanie, c'est un peu de voir ensemble où est-ce qu'on s'en va par rapport au téléphone de ta mère... En ce moment j'peux pas te promettre que j'vais la voir, j'peux pas t'promettre que j'la verrai pas. Même chose pour ton père, tes frères ou tes sœurs si t'en as. Tu vois c'qu'on est en train de faire cet après-midi, c't'une entrevued'exploration. On est en train d'explorer. On est comme des exploratricestoi pis moi. Ça veut dire que chacun de notre bord on "check la gaffe". Toi t'es en train de t'dire " qu'est-ce que c'est que toute cette bebellelà.» Pis t'as ben raison. Pis
En plus de croire la jeune fille lorsqu'elle parle, l'intervenante répond directement à ses questions. Elle ne les retourne pas contre elle pour lui faire admettre qu'elle a des problèmes, par exemple: "Pourquoi toi, penses-tu que c'est toi et non tes parents qui es ici ? Parlons plutôt de toi là-dedans". Au contraire, elle reconnaît que ces questions doivent être posées et qu'elles doivent obtenir réponse pour que Mélanie puisse décider ce qu'elle veut - si effectivement elle veut quelque chose - de l'intervenante dans le cadre spécifique de ce rapport d'intervention. Sans faire de fausses promesses pour gagner la confiance de la jeune fille, l'intervenante rend explicites les règles qui vont régir leur relation. Certaines d'entre elles ne pourront pas être changées (de signaler ou pas une situation d'inceste, par exemple) et il est bon que Mélanie le sache dès le début. Elle ne lui donne pas l'illusion d'un pouvoir qui n'existe pas. 
moi aussi, de mon côté,j'essaie de comprendre c'qui se passe. C'quej'peux faire avec toi, c'que j'peux pas faire. Parce que tu vois moi j'travaille pour une agence qui a des règlements, qui offre certains services pis pas d'autres. Cet après-midi on va juste essayer d'voir sit'esà la bonne place avec la bonne personne. Si c'est non, ben on r'gardera s'y a quelqu'un d'autre que tu veux voir, supposons que tu voudrais voir quelqu'un. C'est-tu clair c'que j'dis? Qu'est-ce que tu penses de tout ça toi?

M. Ben qu'est-ce que moij'supposée d'faire dans tout ça, moi ? Ça va-tu être commeà la maison... J'te dis quelqu'chose, tu dis oui, oui, pis après tu fais c'que tu veux. Comme ma mère, a'me dit " Tu sais Mélanie tu peux toujours me parler ", pis j'y parle, pis à fait rien...

I. Fait que t'es en train de dire que j'pourrais t'écouter pis après faire à ma tête c'est ça?

M. Ouain!

I. O.K. C'que j'peux te promettre tout de suitec'est que quand tu partiras d'ici tu vas savoir où t'en es. J'prendrai aucune décision sans t'en avoir parlé avant, pis j'te demanderai ton opinion sur tout. J'te cacherai pas que j'ai un rôle à jouer ici 
Mélanie, pis que j'ai des recommandations à faire... C'est ma job, ça. Maisj'te tiendrai toujours au courant de c'qui s'passe. Si t'es pas d'accord, tu pourras l'dire. Tu pourras mêmel'écrire dans le dossier si tu veux...

M. Qu'est-ce que ça va m'donner d'écrire c'que j'pense moi?

I. Ben, au minimum ça montre que t'espas d'accord avec moi pis c'que j'pense...

M. Pis sij'pas d'accord avecc'que tu décidestu vastu l'faire pareil?

I. La seule décision... (hésite)... J'essaie d'imaginer quel genre de décision j’pourrais prendre aujourd'hui avec laquelle tu pourrais pas être d'accord... La seule décision que nous avons à prendre cet après-mici, c'est d'voir où est-ce qu'on s'en va ensemble...

M. Ouain... mais supposons qu'on continue à s'voir pis que tu prends des décisions quej'sus pas d'accord avec?

I. La seule raison qui pourrait justifier le fait que j'prenne des décisions contre ton gré, c'est si j'pense que t'es en danger. Y'a une loi qui existe pis, en tant qu'travailleuse sociale, j'suis obligée de l'appliquer. À part ça, j'peux vraiment pas voir dans quelle circonstancej'pourrais 
faire quelque chose quand toi tu veux pas. Pis ça, j't'le promets. Si jamaisilfautque j'brise.cette promesse-là,j'te I'dirai pis on en discutera... C'est-tu clair ça, Mélanie?

M. Ouain... Ok... c'est clair...

\section{SILENCE}

I. Y'as-tu une partie d'toi qui voulait venir me voir cet après-midi ?

M. Ben, j'sais pus quoi faire. C'est vraiment pus vivable chez nous. J'sus tannée de toujours m'faire crier après... J'ai juste 14 ans... j'ai pas d'argent... j'ai rien pour vivre...!

I. Vas-tu à l'école?

M. Des fois...

I. Travailles-tu?

M. Non.

I. Fait que t'as vraiment rien derrière toi. C'est comme si t'étais prise dans une cage. C'est-tu ça?

M. C'est ça. Des fois, j'medisquej'vaism'trouver un chum pis tout sacrer ça là...

I. T'aimerais ça partir d'la maison?

M. C'est peut-être pour ça que mon père y veut pas que j'voye des gars... dire?

I. Qu'est-ce que tu veux

M. Ben, y’a peur que j'parte. Y'aura pus son esclave.

I. Dans quel sens es-tu son esclave?
Maintenant que les règles sont claires et qu'elle a pu exprimer la colère et la rancune que suscitait chez elle cette visite obligée chez la "t.s.", Mélanie est beaucoup plus en mesure de décider si cette personne assise en face d'elle peut ou non lui apporter une ressource dont elle a besoin. C'est ce que l'intervenante va lui demander de faire maintenant. Dans les deux passages qui suivent, elle négocie ouvertement les paramètres du travail qu'elle et Mélanie peuvent faire ensemble dans le cadre du contexte qui a été identifié. 
M. Ben, j'serai pu là pour faire le ménage pis... Pis, y'aura personne pour crier après... et traiter comme d'la marde...

I. Ça semble pas avoir été facile pour toi eh, Mélanie ? J'peux mieux comprendre pourquoi t'avais pas tellement l'goût d'venir me voir aujoud'hui. Tu devais te demander c'que j'allais faire dans tout ça eh?

M. Y’a rien à faire de toute façon : j'sus poignée. J'sais pus quoi faire. Toi, tu peux-tu faire quelque chose?

I. Ça dépend. Qu'estce que tu veux dire par "quelque chose"?

M. Ben, tu peux-tumaider à sortir de là ?

I. Tu veux que j't'aide à quitter la maison, c'est ça ?

M. J'aimerais ça... mais j'ai peur.

I. T'aimerais ça qu'on regarde ça ensemble?

M. Oui.

I. Quitter la maison. C'que ça prend, c'que t'as besoin pour quitter la maison... C'est ça ?

M. Ouain...

I. Veux-tu revenir me voir Mélanie?

M. Ben... ouain...

I. T'hésites encore, eh ?

M. Ben j'sais pas dans quoij'm'embarquelà... C'est un peu épeurant...
L'intervenante croit la jeune fille lorsqu'elle parle de son expérience ("on m'crie toujours après. On décide tout à ma place... »). Elle n'essaie pas, comme on le fait souvent, d'interpréter ces propos afin de trouver la «bibitte noire", la "variable explicative " chez la personne qui cause le problème. Elle voit plutôt dans ces propos un autre témoignage de la difficulté que peut représenter le fait d'avoir 14 ans et d'être une fille dans une société comme la nôtre.

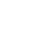


I. T’as raison Mélanie. C'est vrai que c't'épeurant de venirici, mais c't'épeurant aussi d'avoir l'impression d'être prise dans une cage, eh ? Pis j'ai comme l'impression, à t'voir aller, que c'est pas la première fois que tu t'retrouves dans une situation épeurante pis que t'as pas mall'habitude de t'tirerd'affaire... vrai...

M. Ouain... c'tun peu

I. Écoute, supposons qu'on s'entend pour s'rencontrer une couple de fois pour voir si j'peux t'aider à obtenir les choses dont t'as besoin pour prendre ta décision de quitter ou pas la maison. Pis là on r'gardera si I'fait que tu viennes icit'aide dequelque façon que c'soit. Si t'as des plaintes à m'faire, tu m'les fais. Si moi j'en ai, j't'les fais. Sit'es pas d'accord avec c'que j'dis ou fais, tu m'le dis. Même chose pour moi. Pis sit'apprécies c'qu'on fait ensemble, tu m'le dis aussi.

Comment ça t'semble tout ça, ça-tu de l'allure? On as-tu un "deal» toi pis moi?

M. Ouain... O.K. tient compte lorsqu'elle établit l'entente de travail avec elle. Elle ne s'attend pas à ce que l'adolescente "embarque" allègrement dans une aventure sur laquelle elle n'a finalement pas beaucoup de contrôle. Elle lui offre plutôt un échéancier de courte durée : elles évalueront, au bout de deux rencontres, les différents aspects de leur travail ensemble. Mélanie devient ainsi le sujet plutôt que l'objet de l'intervention dont elle est, à ce point-ci, la cible. Ceci entraîne pour elle trois conséquences directes.

D'abord, elle bénéficie au maximum du pouvoir que les limites de cette situation peuvent lui offrir. Ensuite elle apprend qu'il peut exister d'autres types de rapports politiques entre les enfants et les adultes que ceux auxquels les rapports sociaux dominants l'ont jusqu'alors habituée. Et finalement, elle peut décider d'aller chercher des ressources dont elle a besoin pour changer sa situation qu'elle qualifie d'invivable. 


\section{Du pain sur la planche}

Deux tâches importantes attendent celles qui veulent intervenir à partir d'une perspective féministe: développer un discours sociopolitique qui se conjugue au quotidien et créer des pratiques fidèles aux données de l'analyse.

\section{Un discours au quotidien}

Les discours qui décrivent actuellement les formes d'oppression et d'exploitation exercées contre les femmes à l'intérieur des rapports patriarcaux et capitalistes présentent souvent une lacune importante. Ils omettent de décrire également les formes de résistance au quotidien auxquelles donnent naissance ces mêmes rapports sociaux. Assez paradoxalement, ceux-là rebutent et découragent souvent les femmes qu'ils se proposent de " conscientiser » et de « mobiliser ». Ces dernières disent ne pas se reconnaître dans les tableaux noirs que nous leur peignons et nous accusent de ne pas décrire la réalité telle qu'elles la vivent au quotidien. Nos discours leur sont d'autant moins sympathiques que l'idéologie dominante leur en propose des beaucoup plus optimistes pour la bonne raison qu'ils ont l'avantage de simplifier la réalité en offrant du même coup un espoir beaucoup plus à la portée des gens à qui ils s'adressent : celui de croire qu'un changement personnel et intrapsychique va miraculeusement, à lui seul, tout régler.. À titre d'exemple: les tensions et les difficultés que vivent en ce moment les femmes dans leurs rapports avec les hommes sont dues à des causes beaucoup plus complexes que leur unique manque d'amour et d'estime de soi comme le prétendent " ces femmes qui aiment trop ". ${ }^{12}$ Comment une femme enfin "guérie " peut-elle envisager de quitter cet homme qu'elle aime trop et mal lorsqu'elle n'a pas de moyens financiers, pas de travail, peu de support affectif et qu'elle a, par surcroît, la garde entière de trois enfants? De plus, combien de temps peut durer cette estime de soi, sans qu'il existe des conditions pour la nourrir ? En taisant l'importance que jouent la présence ou l'absence de ces ressources dans les façons qu'ont les femmes de se sentir, de penser et d'interagir avec les autres, ce genre de discours les amène à rechercher uniquement en ellesmêmes et/ou chez leurs proches la cause des insatisfactions qu'elles vivent. À court terme, ces explications peuvent sécuriser parce qu'elles leur fournissent un support et une aide tangible pour comprendre et décortiquer ce qui leur arrive. À long terme, par contre, elles contribuent à reproduire les règles du système en leur servant des explications 
personnalistes et psychologisantes qui les empêchent de lutter pour obtenir les ressources dont elles ont besoin pour questionner ces règles et contribuer à les changer. Règles qui rendent en ce moment les relations entre hommes et femmes si difficiles à vivre, peu importent les caractéristiques particulières des personnes en place.

En voulant dépeindre la toile de fond sur laquelle elles doivent vivre actuellement et les difficultés qui en découlent, nos discours féministes ont par contre souvent négligé de souligner que, malgré cette toile de fond et fort probablement à cause d'elle, les femmes ont développé des forces et des formes de résistance qui leur permettent la plupart du temps de tenir le coup et de donner un sens à leur vie. Ces discours parfois teintés de déterminisme, arrivent tant bien que mal à expliquer pourquoi tant de femmes ont "de la misère». Ils arrivent beaucoup moins bien, par contre, à expliquer pourquoi tant de femmes, sous ces mêmes rapports sociaux, arrivent aussi et quand même à aimer leurs hommes et leurs enfants, à travailler, à rire, à dormir, à s'amuser, à ne pas prendre de pilules, à faire l'amour... à vivre quoi ! Ils arrivent mal aussi à expliquer pourquoi, sous ces mêmes rapports sociaux, certains hommes sont corrects dans leurs relations avec les femmes et les enfants.

Le défi de développer un tel discours est de taille car, ce faisant, nous allons à l'encontre des explications déterministes et volontaristes qui dominent en ce moment au sein de notre société. Mais comme nous avons cherché à le démontrer, ces explications nous piègent en nous rendant, soit coupables, soit impuissantes... Alors, avons-nous vraiment le choix?

\section{Une pratique fidèle à l'analyse}

Celles qui veulent intervenir à partir d'une perspective féministe doivent également s'attaquer à une seconde tâche importante : traduire leurs analyses dans les termes concrets d'une pratique.

La plupart des modèles d'intervention non féministes tendent à placer le problème d'abord et avant tout chez les personnes en cause et aboutissent tous, de façon plus ou moins implicite, à blâmer les gens pour les difficultés qu'ils rencontrent dans leur vie de tous les jours. ${ }^{13}$ Ceci donne naissance à des pratiques dont l'objectif final est de changer la personne, soit au niveau de son comportement (thérapies behaviorales et groupes d'affirmation de soi), de ses sentiments (gestalt, feeling therapy), de ses idées (restructuration cognitive et développement émotivo-rationnel), de son corps (bio-feedback, bio-énergie) et même 
de ses niveaux de conscience (certaines pratiques de " conscientisation "). Puisque les praticiennes féministes ont souvent été formées à ces différents modèles et tendent à en emprunter les techniques et les habiletés, il existe un véritable danger qu'elles adoptent aussi, sans trop s'en rendre compte, les analyses qui les sous-tendent et, ce faisant, tiennent encore une fois les femmes responsables des situations difficiles dans lesquelles elles se retrouvent. Par exemple, plusieurs des pratiques développées depuis quelques années pour venir en aide aux femmes violentées s'appuient fortement sur le modèle behavioriste et ont comme but final de modifier les comportements de victimes que les femmes ont appris très jeunes. Même si les théoriciennes de ces pratiques disent tenir compte de facteurs socio-politiques, l'emphase qui est mise sur les techniques d'affirmation de soi comme moyen d'intervention privilégié laisse quand même sous-entendre que les femmes sont battues parce qu'elles manquent d'estime de soi et de confiance en elles. Un peu comme si on leur disait : "Si t'adoptais moins des comportements de victime, tu serais moins battue... ». De telles explications négligent de tenir compte du fait que les comportements de ces femmes - si passifs soient-ils - ne sont pas la cause du problème. La violence faite aux femmes n'est pas un phénomène behavioral mais bien socio-politique qui prend racine à même le patriarcat. Comme nous l'avons mentionné à plusieurs reprises, beaucoup de femmes sont privées des ressources dures et molles dont elles ont besoin pour quitter ou transformer une situation de violence. Le seul, l'unique but que peut avoir une intervention féministe dans un tel contexte, c'est celui d'obtenir à court, à moyen et à long terme le plus grand nombre possible de ces ressources.

Ceci ne veut pas dire que nous sommes opposées à travailler avec les femmes pour les aider à changer certains de leurs comportements, de leurs pensées, etc. Mais en ceci, notre modèle diffère des autres de deux façons. D'abord parce que nous donnons aux femmes et aux filles en face de nous le pouvoir de décider quels comportements, quelles pensées elles veulent changer, et celui de nous dire quel va être notre rôle d'intervenante là-dedans. Ensuite, parce que travailler à changer des facettes d'une personne n'est jamais le but de notre intervention. Nous aidons les femmes à changer, en autant qu'il est clair entre nous à quelles ressources ces changements vont donner accès. Par exemple, nous pourrions travailler sur la confiance en soi s'il est clair au départ que cette confiance en soi peut contribuer, mais jamais suffire, à elle seule, à modifier une situation de violence. De tels changements peuvent, à court terme, aider à obtenir certaines ressources molles, par exemple exprimer un besoin de tendresse à un groupe de femmes; 
mais ils mènent rarement à l'obtention des ressources dures sur lesquelles l'existence même des ressources molles se fonde. Par exemple, l'amour de soi peut être un moyen important si ce que l'on vise est de créer une relation plus intime avec une autre personne. Par contre, cet amour de soi ne suffira pas lorsque viendra le temps d'obtenir un travail valorisant ou un revenu décent. Et sans la présence de ces deux ressources, l'amour de soi si difficilement acquis en thérapie risque de s'évaporer à plus ou moins courte échéance si vous devez vivre sur le bien-être social dans une société telle que la nôtre...

Le défi de développer des pratiques qui tiennent compte des données de l'analyse féministe est lui aussi de taille. Confrontées de toutes parts à des modèles qui reproduisent l'idéologie dominante et les rapports sociaux qui les sous-tendent, en tenant les femmes encore une fois responsables et donc, d'une certaine manière, toujours coupables de leur sort, les intervenantes se retrouvent devant un quasi-vide méthodologique difficile à combler parce que cela implique d'aller à contre-courant de presque tout ce qui se fait en ce moment. Seules à seules nous n'y arriverons pas. Cet article a la prétention de lancer une perche qui nous permettra de nous en parler...

\section{En guise de conclusion}

Les hypothèses de travail que nous venons de présenter sont le fruit et le produit de nombreuses conversations que nous avons eues avec des praticiennes et des praticiens, des collègues universitaires, des fonctionnaires et des "bénéficiaires" de nos services (mais peut-on toujours prétendre qu'elles en bénéficient ?). En présentant ces hypothèses, nous ne prétendons pas qu'une nouvelle façon d'analyser les difficultés auxquelles ont à faire face les femmes et d'y intervenir peut à elle seule enrayer le burn-out chez les intervenantes, dont nous parlions au début de cet article. Sous-entendre une telle chose équivaudrait à tomber encore une fois dans la tendance dominante qui, même si elle dit reconnaître l'impact de certaines tensions structurelles, finit toujours par subtilement blâmer les intervenantes pour l'état actuel des pratiques en travail social. ${ }^{14}$ Dans ce cas-ci, ce blâme se résumerait comme suit : "Si t'avais juste une meilleure analyse et une meilleure façon d'intervenir, tu ne te brûlerais pas comme tu le fais". Et l'intervenante de se retrouver, en tant que femme, avec ce sentiment qu'elle connaît si bien : la culpabilité, l'arme suprême, comme nous l'avons dit plus tôt, de l'impuissance et de la soumission... 
Nous croyons, par contre, que l'approche que nous présentons peut contribuer à contrecarrer cette idéologie psychologisante et individualiste qui, en renvoyant toujours les intervenantes à leurs propres lacunes, les empêche de questionner et de transformer les rapports sociaux qui les engendrent.

Survivre sur la corde raide en talons hauts est certes une opération périlleuse, mais cela peut devenir stimulant et excitant pour celles qui ont accès à des ressources qui leur permettent d'enlever ces souliers maudits, d'oublier leurs pieds et de fixer les yeux droit devant elles.

\section{Notes}

* L'auteure tient à remercier Nancy Guberman et Alain Novel pour leur contribution à la rédaction de cet article.

1 Il est ici question de ressources " dures " et "molles ". Par ressources " dures", nous entendons les ressources matérielles plus évidentes telles que l'argent, la nourriture, le logement, mais également celles du genre services médicaux non sexistes, contraception et avortement libre, gratuit et accessible, salaire égal pour travail égal, rues sans danger, etc. Par ressources "molles", nous faisons référence aux ressources affectives et émotives telles que l'attention, le respect, la reconnaissance sociale, l'estime de soi, l'affection des autres, etc. Dans notre société, la présence des ressources « molles » dépend plus souvent qu'autrement de la présence de ressources "dures".

2 Cette recherche fait le compte rendu et l'analyse des propos de douze expertes dans des domaines tels que l'immigration, la syndicalisation, l'éducation des adultes, les communications, les nouvelles technologies de la reproduction, la politique municipale, provinciale et fédérale, la démographie. Ces femmes ont été interviewées par des intervenantes du Y.W.C.A. afin de connaître leurs prévisions des conditions de vie et de travail des Montréalaises de l'an 2000.

3 Pour une vision critique de l'état actuel des média, voir l'excellent livre de Nicole Beauchamp paru en 1987 (cité en bibliographie).

4 Tous les termes présentement utilisés pour désigner le "sujet du travail social " (Laforest et Redjeb) nous laissent insatisfaites car ils cachent tous la nature politique et idéologique qui existe entre l'intervenante et la ou les personnes devant elle. Aucun des termes utilisés n'est neutre; ils appartiennent tous à une des visions dont il sera question au point suivant. Par exemple, le terme " usager ", très populaire en ce moment, est un mot qui découle de la vision technocratique de la pratique. Pour faciliter la compréhension de notre texte et en même temps contourner ce choix de termes, nous avons choisi de mettre des guillemets lorsque nous utilisons soit un terme soit un autre.

5 Très schématiquement, il existe actuellement trois média-visions du travail social qui prennent forme dans des discours très contradictoires sur la nature et la fonction de la pratique : le discours technocratique tenu par plusieurs des fonctionnaires du M.S.S.S. et par de nombreux cadres de C.S.S., C.L.S.C. et C.A. ; le discours professionnel que l'on retrouve surtout chez les représentantes 
de la Corporation professionnelle des travailleurs sociaux et chez les cadres intermédiaires des milieux de pratique; et le discours militant, plus présent chez les intervenantes et intervenants de la base. Pour une description plus détaillée de ces trois types et de leurs composantes respectives, voir les articles de Pierre Racine, de Lionel Groulx et un texte inédit de l'auteure (cités en bibliographie).

6 Christina Maslach donne la définition suivante du burn-out professionnel: " une situation d'épuisement physique et émotionnel, impliquant le développement d'attitudes négatives au travail et de perceptions elles aussi négatives, un concept de soi médiocre comme professionnel et la perte d'un intérêt empathique pour le client auquel divers services sont offerts ". (Traduction de Néré Saint-Amand). D'après ce dernier, plus de $50 \%$ des nouveaux gradués connaissent cet état de burn-out après seulement deux ans de pratique.

7 Ce contexte est décrit de façon éloquente dans l'article de Camil BouCHARD : "Intervenir à partir de l'approche écologique : au centre, l'intervenante ", qui paraît dans ce numéro.

8 Pour une critique plus détaillée de la notion d' "individu " et une définition plus complète de celle d' "acteur social ", voir l'article de Frédérik Mispelblom cité en bibliographie.

9 Puisque cet homme adopte des comportements et des attitudes que nous jugeons " corrects", il serait intéressant de savoir à quelles ressources il a eu et a accès pour penser et agir de la sorte...

10 Ces trois visions du rapport (i.e. d'aide, d'égalité et de service) découlent des méta-visions dont nous faisons mention au point 5 . Elles sous-entendent des conceptions très différentes sur la nature et la fonction du travail social.

11. La version anglaise de cette entrevue paraît dans "How feminism can take the crazy of your head and put it back into society ", cité en bibliographie.

12 C'est le titre du best-seller de Robin Norwood, qui fait présentement des ravages au Québec. Comme la plupart des livres du même genre - et ils apparaissent à un rythme effrayant depuis quelques mois - celui-ci mise sur l'insatisfaction profonde que vivent les femmes actuellement et séduit par l'apparente simplicité des solutions proposées : apprendre à s'aimer.

13 Pour une critique intéressante des divers modèles présentement répandus, voir entre autres l'excellent livre de Joel Kovel. Voir également l'article de Maurice Moreau sur les modèles de thérapie familiale et le livre Psychothérapies Attention! paru en 1983 (cités en bibliographie).

14 Pour un bel exemple de ce genre de blâme subtil, voir le texte de la présentation de Madame Jeanne d'Arc Vaillant, alors sous-ministre au M.S.S.S., lors de la conférence interrégionale de la Corporation professionnelle des travailleurs sociaux, à Québec, en 1983. 


\section{Bibliographie}

Beauchamp, Nicole, Le silence des média, Montréal, Remue Ménage, 1987.

Bouchard, Camil et al, Psychothérapies, Attention !, Québec, Presses de l'Université du Québec, 1983.

Bourgon, Michèle et Nancy Guberman, "How Feminism Can Take the Crazy out of your Head and Put it Back into Society ", dans: Women's Studies: A Canadian Perspective, Vancouver, Bergammon Press, printemps 1988.

BouRGON, Michèle, Rapport et analyse d'entrevues menées dans le cadre de la recherche sur les besoins des montréalaises de l'an 2000, texte polycopié, Département de travail social, UQAM, 1987.

BourGon, Michèle, Vision conflictuelle en travail social contemporain au Québec, (inédit), Département de travail social, UQAM, 1986.

Groulx, Lionel, "Discours technocratique en service social », Le travailleur social vol. 48, no 4, 1980: 183-187.

Groulx, Lionel, "Discours féministe en travail social : analyse et interprétation ", Revue canadienne de service social, 1985 : 123-137.

Kovel, Joel, A Complete Guide to Therapy: From Psychoanalysis to Behavior Modification, New York, Pantheon Books, 1976.

LAFOREST, Marcelle et Belhassen REDJEB, " Analyse de l'intervention technocratique dans les services sociaux au Québec; Revue canadienne de service social, 1983: 106-128.

MASLACH, Christina, "Burned-out ", Human Behavior, vol. 5, no 9, 1976 : 16-22.

MASLACH, Christina, "Job Burnout: How People Cope", Public Welfare, vol. 36, n० 2, $1978: 56-58$.

MisPelbloM, Frédérik, "Pratiques "socio-historiques" en travail social», Revue d'action communautaire, no 8/48, 1982 : 177-189.

MOREAu, Maurice, "L'approche structurelle familiale en service social ", Revue d'action communautaire, no 7/47, 1982: 159-170.

Norwood, Robin, Ces femmes qui aiment trop, Montréal, Stanké, 1986.

RACINE, Pierre, Les relations d'autorité : réflexion sur une évolution dans leur usage professionnel en travail social au Québec, Département de travail social, UQAM, 1987.

SAINT-AMAND, Néré, "Stress et épuisement professionnel: implication pour la formation en travail social», Revue canadienne de service social, 1985 : 138-153.

VAILLANT, Jeanne-D'Arc, La pratique sociale de demain au Québec, texte polycopié de la conférence prononcée au Congrès interrégional de la Corporation professionnelle des travailleurs sociaux, Québec, le 21 octobre 1983. 